\title{
An Innovative Approach to Authenticate a Person using Mouse Gesture Dynamics
}

\author{
C.A. Anupashree, Spoorthi S. Kulkarni and Parashuram Baraki
}

\begin{abstract}
A unique method for biometric authentication is through the mouse gesture of an individual, though this technique, the movement of mouse as a pointing device is assessed, two types of authentication are recognized, static and continual. The most prominently utilized system is continual. Static authentication has been slow to develop whereas continual systems have rapidly evolved. To solidify effectiveness of authentication, we have introduced a new model which is robust. In this approach, user draws a gesture using mouse. These gestures are collected and evaluation through a cover markov model classifier, a remarkable consistency in mouse gesture systems, in regards to False Rejection Ratio and False Acceptance Ratio in comparison to conventional systems. From the results, one can observe that there is improvement in terms of precision and authentication in comparison to conventional systems. The report concludes, as we believe the first time in history, that a mouse gesture system successfully recognized its purpose accurately.
\end{abstract}

Keywords--- Component Behavioral Biometric, Mouse Dynamics, Mouse Dynamics Analysis Framework, Data Acquisition, Data Preprocessing.

\section{INTRODUCTION}

$\mathrm{T}$ HE primary point of developing biometric system is to convey precise and effective authentication.. Mouse gesture dynamics is considered to a new behavioral biometric example[1], this approach has gained considerable prominence recently. Mouse dynamics biometric technology is gaining importance given its capability of monitoring consistently a computers' usage. This text recognizes the users' attributes or fluctuations when a user utilizes the mouse. This leads to the creation of mouse gestures. Whenever user enters into a session his credentials are checked and the user is authenticated. Mouse gesture is drawn by considering data points with coordinate values. During the registration phase, mouse gestures will be conducted over the computer screen by the user multiple times. The movement is registered and subsequently assessed through relevant systems. Later on, this collected data is utilized for authentication of the user. During the authentication phase, individuals will require to perform identical mouse gestures as they were performed in the registration phase.Syurki has suggested utilization of a signature, drawn by users as part of the recognition procedure whereas the suggestion of Revetter utilizes mouse locking for static recognition. Each performed gesture is assessed for recognition. .Fig1 depicts drawn gestures from individuals at computer login through time with 14 data points.

C.A. Anupashree, UG scholar, Dept. of CSE, GMIT Davangere, Karnataka.

Spoorthi S. Kulkarni, UG scholar, Dept. of CSE, GMIT Davangere, Karnataka.

Parashuram Baraki, Asst. Professor, Dept. of CSE, GMIT, Davangere, Karnataka.

DOI:10.9756/BIJSESC.8271

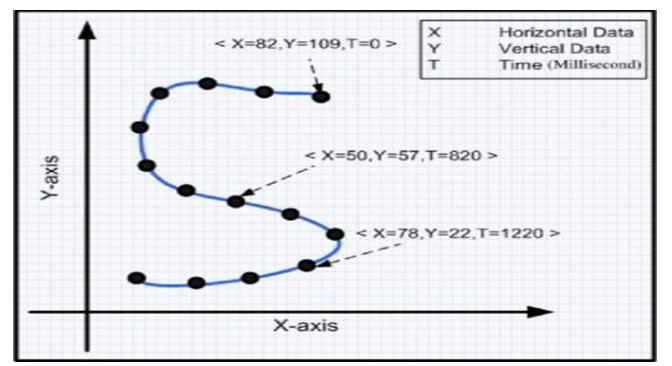

Figure 1: Gesture Involving $n=14$ Data Points

\section{SYSTEM DESIGN}

Figure 2 depicts authentic flow diagram of collection and evaluation of gestures. This method incorporates individuals to perform multiple gestures in repetition[2]. Given that the new data stemmed from these gestures is a match with the saved sample, which had been collected during registration phase. Information obtained through the drawing vicinity (Gesture drawn in fig1) incorporate horizontal (x-axis) and vertical coordinate(y-axis)and spanned period in milliseconds for every individual pixel. Data points are sequenced for gesture or pattern replication, with each point being signified through a triplet $\langle\mathrm{x}, \mathrm{y}, \mathrm{t}\rangle$ indicating $\mathrm{X}$-coordinate, $\mathrm{Y}$ coordinate and elapsed time respectively. Jth duplication of a gesture $G$ is signified as a sequence $G j=\{\langle x 1 j, y 1 j, t 1 j\rangle,\langle x 2 j, y 2 j, t 2 j\rangle,\langle x n j$, nj,tnj>\}, where $n$ refers towards gesture size(GS) and each $<x i j, y i j, t i j>$ where $(1 \leq \mathrm{i} \leq \mathrm{n})$ is recognized as data point. The primary objective is to recognize variations between individuals' on their behavioural biometrics while drawing patterns[3].

\section{MOUSE PATTERn ASSESSMENT SySTEMS INCORPORATE THE MENTIONED MODULES}
A. Gesture creation module.
B. Data acquisition and preparation module.
C. Feature extraction module.
D. Classification module.

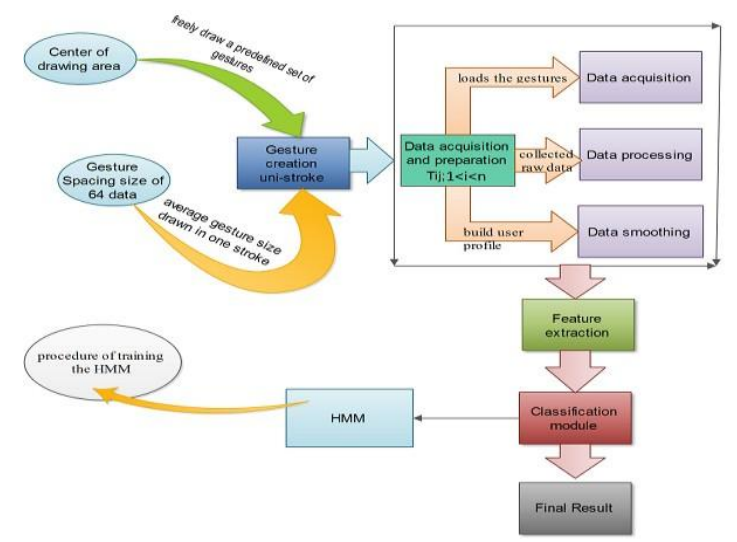

Figure 2: Flow Diagram for Capture and Analysis of Mouse Gestures 


\section{A. Gesture Creation Module}

The development module is basic drawing software which collects the users' gestures as they use the mouse. There is no specific pattern of language that the collected patterns are organized into, and neither do the gestures have any particular meaning. There are three parameters to consider for each pattern, horizontal, vertical and elapsed millisecond timing.

\section{B. Feature Extraction Module}

This module derives characteristics from unprocessed data. Feature choosing is facilitated through assessing template data and recognizing features. Extracts from vectors that are seized amidst two mouse clicks can be utilized. The finished list of obtained features is detailed under Table.

Table1 : Feature Extracted From Raw Data

\begin{tabular}{l|c}
\hline Feature Description & Notation \\
\hline Horizontal coordinate & $x$ \\
Vertical coordinate & $y$ \\
Absolute time & $t$ \\
Horizontal velocity & $v v$ \\
Vertical velocity & $t v$ \\
Tangential velocity & $t a$ \\
Tangential acceleration & $t j$ \\
Tangential jerk & $l$ \\
Path from the origin in pixels & $\theta_{i}$ \\
Slope angle of the tangent & $c$ \\
Curvature & $\delta c$
\end{tabular}

\section{EXPERIMENTAL RESULTS}

This section presents the experimental assessment of the suggested system. The frame work consists of two phases for authentication. Registration is the first phase in which the user is asked to register his/her name by drawing gesture using Mouse [5]. The user is allowed to draw any type of gesture which may include alphabets type or numeric type or combination of both or any other type of symbols. The gesture will be stored in the database for future reference. The gesture is then trained to understand the input given by the user.

Following snapshot shows the process involved in Registration phase. In this phase user is required to register along with their name by drawing their own gesture in the given window. Soon after this system will be trained and it will shown the display the gesture drawn by user on display window.

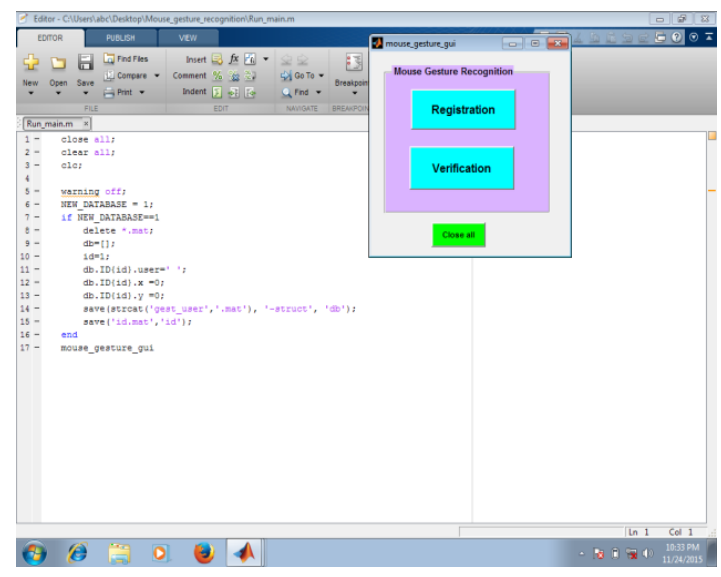

Figure 3: Mouse Gesture Main Window

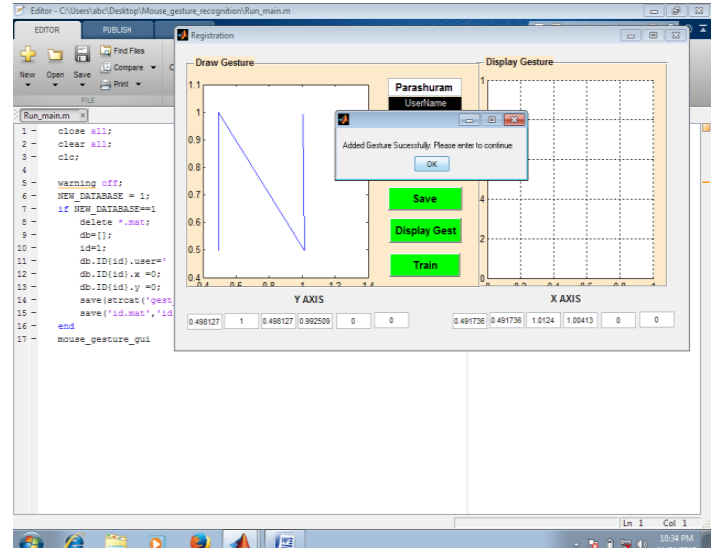

Figure 4: Shows Method of Adding Gesture Along with User Name

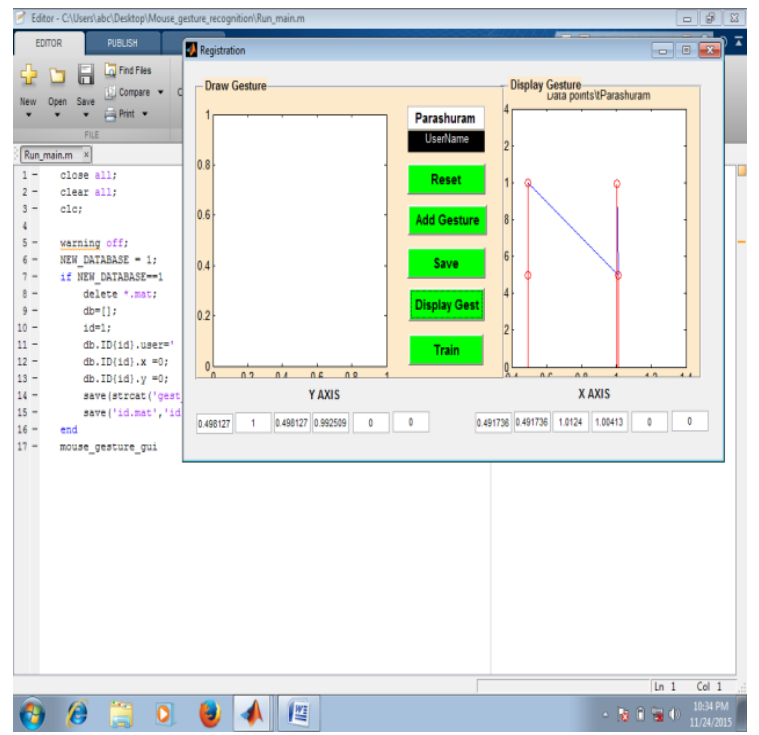

Figure 5: After Training, Gesture will be Displayed on Display window

After registration phase, the system will determine whether the user is valid or invalid user using Verification phase.

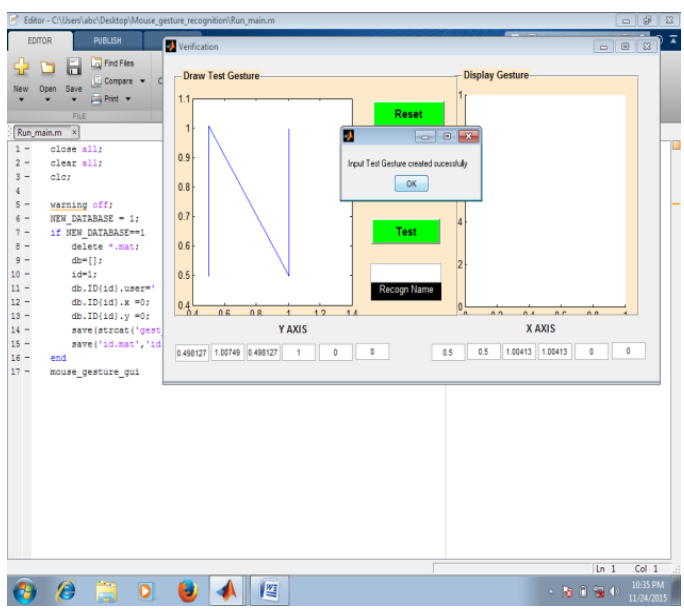

Figure 6: Gesture Drawn by user for Verification 


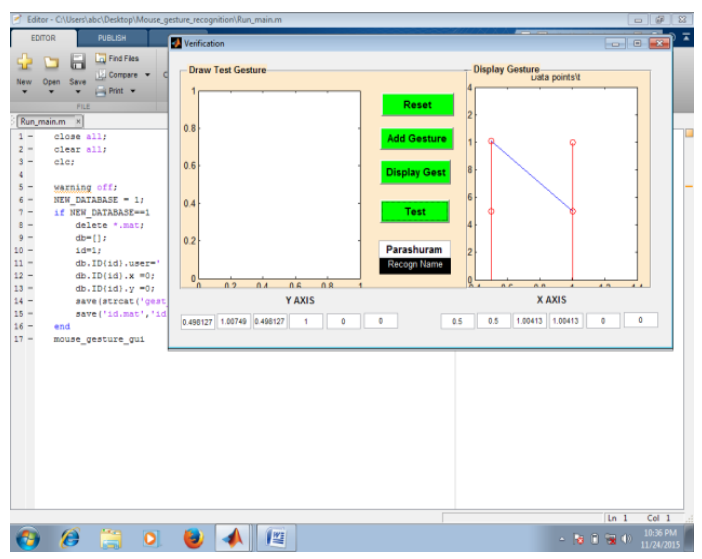

Figure 7: After Selecting Test, If Gesture Matches then it Recognizes Authenticated User and Displays Name of User.

The above snapshots show the result of valid authentication. If the user is valid, his/her name will be displayed on the window soon after the drawn gesture matches with gesture stored in database. If gesture does not match then "Unknown user" will be displayed. Following snapshot represents the failure case.

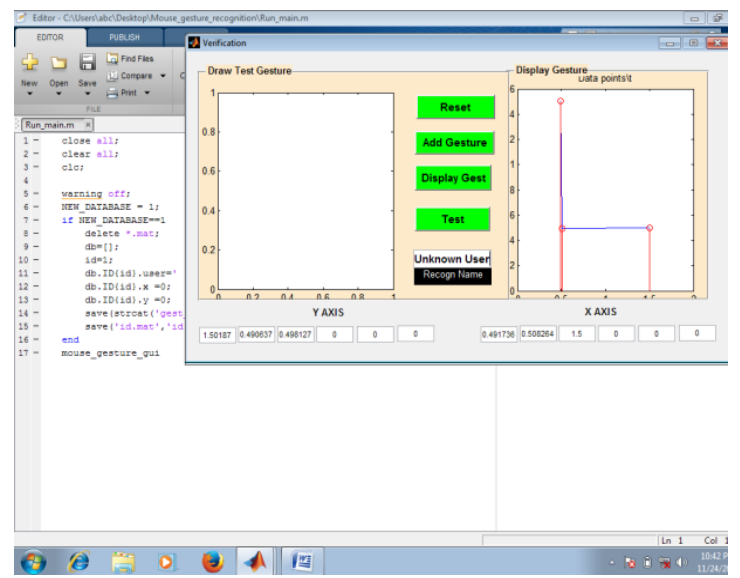

Figure 8: When Gesture does not Match then user will be Considered as Unknown User

\section{Advantages}

1. An accelerometer-based digital pen for handwritten digit and gesture trajectory recognition applications.

2. The system is intuitive, and tracing the naturalness of the handwriting proves to be of excellent acceptance.

3. Information is traceable back to a specific pen and data is time-stamped for added $\neg$ accountability.

\section{GRAPHICAL COMPARISON OF EXISTING AND PROPOSED WORKS}

The proposed algorithm yields better results compared to the existing system. We have considered 17 different gestures drawn by user using Mouse and achieved an efficiency of $78 \%$.

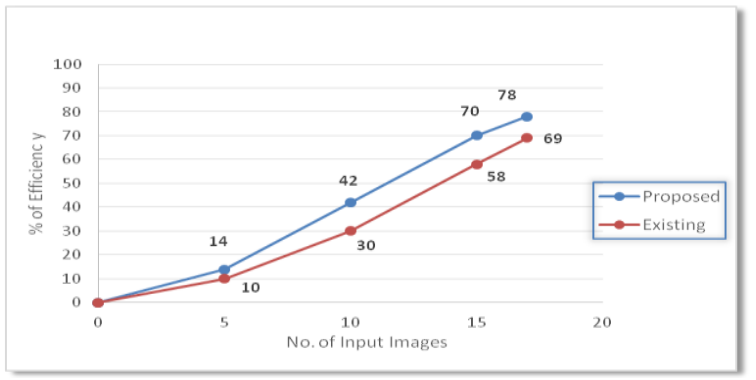

Figure 9: Graphical Representation of the Result.

Conclusion

In this work, we have considered authentication of the user using dynamic mouse gesture. Results demonstrate that the proposed algorithm reliably recognizes users with higher recognition rate when compared to existing methods. The result obtained confirms the higher security in the biometric authentication system.

\section{REFERENCES}

[1] B. Sayed, I. Traore, I. Woungang and M.S. Obaidat, "Biometric Authentication Using Mouse Gesture. Dynamics", IEEE systems journal, Vol. 7, Pp. 262-274, 2013.

[2] S. Climate, M. Gamassi, V. Piuri, R. Sassi and F. Scotti, "Privacyaware biometrics: design and implementation of a multimodal verification system," In Computer Security Applications Conference, Pp. 130-138, 2014.

[3] L. Ballard, F. Monrose and D.P. Lopresti, "Biometric Authentication Revisited: Understanding the Impact of Wolves in Sheep's Clothing", In USENIX Security, 2006.

[4] M.S. Obaidat and B. Sadoun, "Verification of computer users using keystroke dynamics", IEEE Transactions on Systems, Man, and Cybernetics, Part B (Cybernetics), Vol. 27, No. 2, Pp. 261-269, 1997.

[5] M. Marengoni and D. Stringhini, "High level computer vision using OpenCV", In Graphics, Patterns and Images Tutorials (SIBGRAPI-T), 2011 24th SIBGRAPI Conference on, Pp. 11-24, 2011.

[6] B. Sayed, I. Traore, I. Woungang and M.S. Obaidat, "Biometric authentication using mouse gesture dynamics", IEEE Systems Journal, Vol. 7, No. 2, Pp. 262-274, 2013.

[7] L. Dignan, "ARM Holdings 2015 Plan: Grab PC", server share, 2011.

[8] G. Bradski and A. Kaehler, "Learning OpenCV: Computer vision with the OpenCV library", 2008.

[9] C.M. Bishop, "Pattern recognition. Machine Learning, 128. 2006

[10] M. Marengoni and D. Stringhini, "High level computer vision using Open CV", In Graphics, Patterns and Images Tutorials (SIBGRAPI-T), Pp. 11-24, 2011. 